\title{
Quantum mechanical relaxation of open quasiperiodic systems
}

\author{
A. Ossipov, M. Weiss, Tsampikos Kottos, and T. Geisel \\ Max-Planck-Institut für Strömungsforschung und Fakultät Physik der Universität Göttingen, \\ Bunsenstraße 10, D-37073 Göttingen, Germany
}

\begin{abstract}
We study the time evolution of the survival probability $P(t)$ in open one-dimensional quasiperiodic tight-binding samples of size $L$, at critical conditions. We show that it decays algebraically as $P(t) \sim t^{-\alpha}$ up to times $t^{*} \sim L^{\gamma}$, where $\alpha=1-D_{0}^{E}, \gamma=1 / D_{0}^{E}$ and $D_{0}^{E}$ is the fractal dimension of the spectrum of the closed system. We verified these results for the Harper model at the metalinsulator transition and for Fibonacci lattices. Our predictions should be observable in propagation experiments with electrons or classical waves in quasiperiodic superlattices or dielectric multilayers. PACS numbers:05.60Gg,03.65Nk,72.15Rn
\end{abstract}

The decay properties of open quantum mechanical systems, have been attracting considerable attention for several decades. Their study was motivated by various areas of physics, ranging from nuclear [1], atomic [2] and molecular [3] physics, to mesoscopics [4] and classical wave scattering [5]. In recent years, the interest in quantum mechanical decay was stirred by mesoscopic cavities and microwave billiards where immediate experimental realizations have become feasible [6]. At the same time various analytical techniques have been developed to study the problem in more detail. One possible formulation of the problem is to consider the survival probability $P(t)$ of a wave packet localized initially inside the open system. In particular it was found that this quantity exhibits slower than exponential decay (i.e. long-time tails) for disordered wires in the localized (in one-dimension) [7] and metallic (in higher dimensions) regimes [8 11]. Moreover, for ballistic systems, Random Matrix Theory (RMT) predicts an algebraic decay $P(t) \sim 1 / t^{\beta M / 2}$, where $M$ is the number of channels and $\beta=1(2)$ for preserved (broken) time reversal symmetry 12,13].

The investigation of the survival probability has recently been extended to quantum systems with a mixed classical phase space [14,15] where it was found that $P(t) \sim 1 / t$. The same algebraic decay was found for systems with exponential localization. In both cases, this law is related to localization and tunneling effects and applies for intermediate asymptotic times [14].

The subject of the present paper is the survival probability in a new setting, namely a class of systems whose closed analogues have fractal spectra. The latter exhibit level clustering [16] in strong contrast to the level repulsion predicted by RMT for systems in the ballistic regime and to the Poisson statistics in the localized regime [17]. Representatives of this class are quasi-periodic systems with a metal-insulator transition like the Harper model [16, 18, Fibonacci chains [16, 19, or quantum systems with a chaotic classical limit like the kicked Harper model [20.

Here for the first time we present consequences of the fractal nature of the spectrum for the quantum time evolution of open systems. In particular, we ask how they are encoded in the survival probability $P(t)$, which is the simplest quantity measured in laboratory experiments. We show that $P(t)$ decays as

$$
P(t) \sim 1 / t^{\alpha} ; \quad \alpha=1-D_{0}^{E},
$$

where $D_{0}^{E}$ is the fractal (box-counting) dimension of the spectrum of the closed system. Moreover, we determine the time scale $t^{*}$ up to which this power-law decay can be observed. It scales as

$$
t^{*} \sim L^{\gamma} ; \quad \gamma=1 / D_{0}^{E},
$$

where $L$ is the sample size. Beyond this time scale $P(t)$ decays exponentially. Our results (11),(2) are confirmed numerically for two types of quasi-periodic tight-binding models and are supported by analytical arguments.

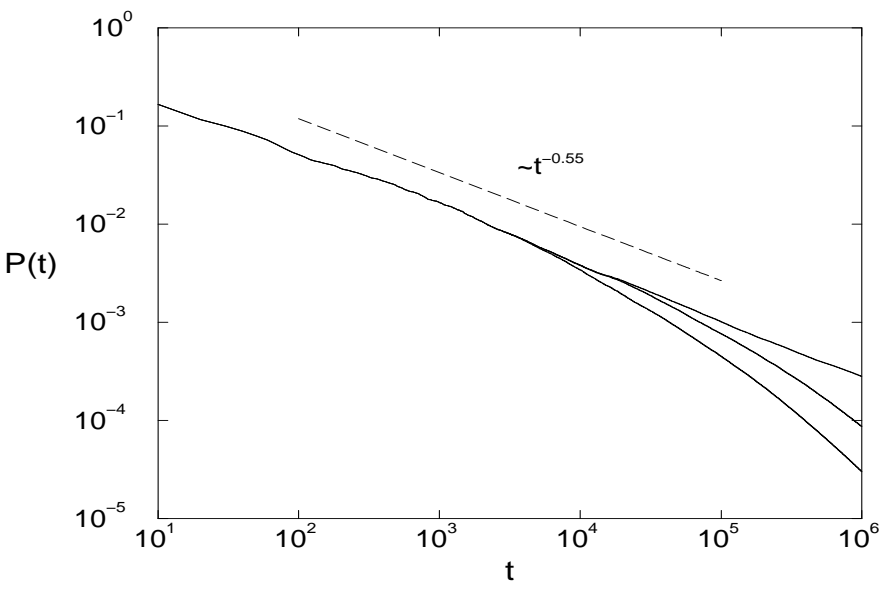

FIG. 1. The survival probability $P(t)$ of the Harper model $(\lambda=2)$, for three different sample lengths $L=250,500,4000$ exhibits an inverse power-law $P(t) \sim t^{-\alpha}$. A least squares fit yields $\alpha=0.55 \pm 0.05$ in accordance with $D_{0}^{E} \simeq 0.5$ and Eq. (㑑).

The mathematical model we consider is the timedependent Schrödinger equation

$$
i \frac{d \psi_{n}(t)}{d t}=V_{n} \psi_{n}(t)+\psi_{n+1}(t)+\psi_{n-1}(t),
$$

where $\psi_{n}(t)$ is the probability amplitude for an electron to be at site $n$ of a one-dimensional (1D) sample 
of length $L$. The on-site potential $V_{n}$ is determined by quasi-periodic sequences. We assume absorbing boundary conditions at the ends of the sample [21] and initial excitations in the form of a $\delta$-like packet launched at one of the boundaries, i.e. $\psi_{n}(t=0)=\delta_{n, 1}$. Equation (3) has been integrated numerically using a Cayley scheme [22] with integration time step $d t=0.1$. We attached 15 additional sites at the ends of the sample and erased all components of the wave packet on these sites after each time step $d t$. The decay of the norm of the wave packet obtained in this way was not affected by a further decrease of $d t$.

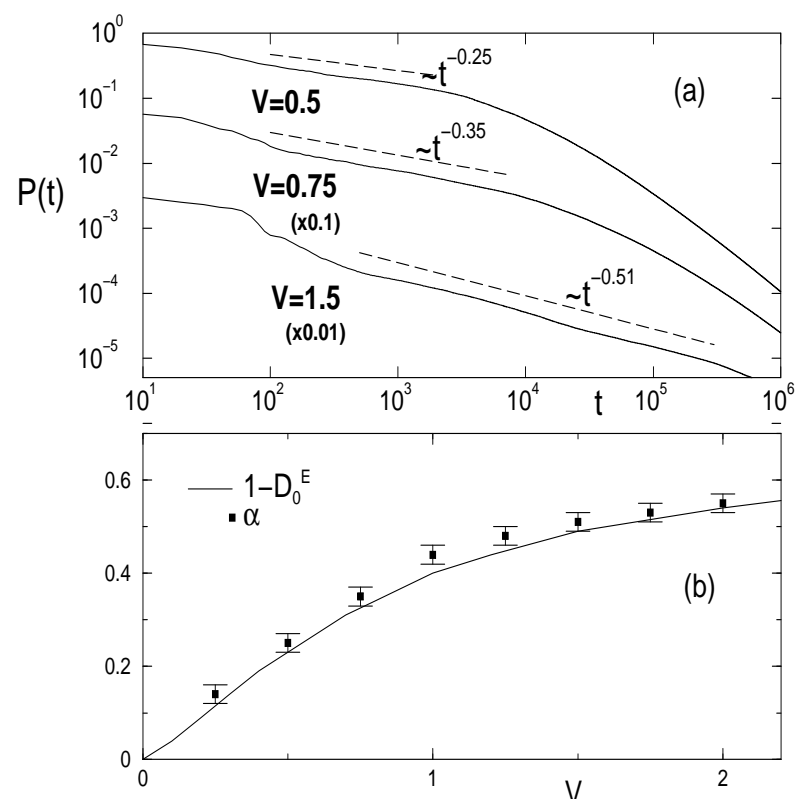

FIG. 2. (a) Survival probabilities $P(t)$ of the Fibonacci model, for three different potential strengths $V_{1}=0.5, V_{2}=0.75$ and $V_{3}=1.5$ showing inverse power-laws $P(t) \sim t^{-\alpha}$ (dashed lines). The sample size is $L=2000$ in all cases. (b) Power-law exponents $\alpha$ of the survival probability obtained numerically as a function of the potential strength $V$ for the Fibonacci model. The solid line is the theoretical prediction $\alpha=1-D_{0}^{E}$.

We motivate and numerically verify our results using first the well known Harper model, a paradigm of quasiperiodic 1D systems with a metal-insulator transition [16,18. It is described by a tight-binding Hamiltonian with an on-site potential given by

$$
V_{n}=\lambda \cos (2 \pi \sigma n) \text {. }
$$

This system effectively describes a particle in a twodimensional periodic potential in a uniform magnetic field with $\sigma=a^{2} e B / h c$ being the number of flux quanta in a unit cell of area $a^{2}$. When $\sigma$ is an irrational number, the period of the effective potential $V_{n}$ is incommensurate with the lattice period. The states of the corresponding closed system are extended when $\lambda<2$, and the spectrum consists of bands (ballistic regime). For $\lambda>2$ the spectrum is point-like and all states are exponentially localized (localized regime). The most interesting case corresponds to $\lambda=2$ of the metal-insulator transition. At this point, the spectrum is a Cantor set with fractal dimension $D_{0}^{E} \leq 0.5$ [23] while self-similar fluctuations of the eigenstates exist on all scales [16, 18].

$\Gamma_{\mathrm{k}}$
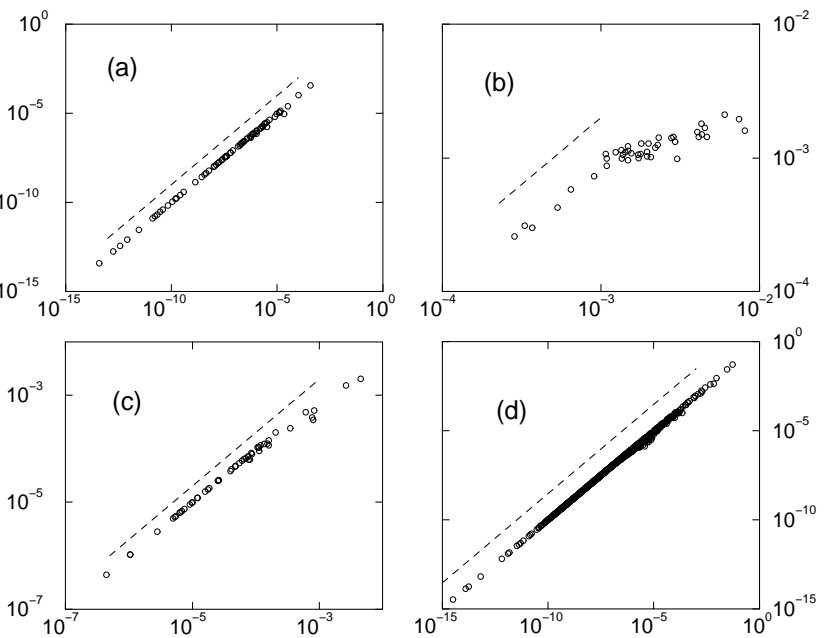

FIG. 3. Resonance widths $\Gamma_{k} \stackrel{\left|c_{k}\right|^{2}}{a s}$ a function of the overlapping elements $\left|c_{k}\right|^{2}$. The data are obtained by direct diagonalization of the effective Hamiltonian $\mathcal{H}_{\text {eff }}$ (see Eq. (6)). The dashed lines of slope 1 are shown for comparison demonstrating a linear relation for small $\Gamma$. In all cases the sample size is $L=1597$, corresponding to an approximant of the golden mean $\sigma=\frac{987}{1597}$. (a) Harper model for $\lambda=2$; (b) Fibonacci model for $V=0.1$; (c) Fibonacci model for $V=0.5$; and (d) Fibonacci model for $V=1.5$.

We investigate the survival probability $P(t)$ for the Harper model at the critical point $\lambda=2$. In our calculations we assume $\sigma$ equal to the golden mean $\sigma_{G}=$ $(\sqrt{5}+1) / 2$. For this case it is known that $D_{0}^{E} \approx 0.5$ 23. The results for various sample lengths $L$ are shown in Fig. 1. In all cases the survival probability clearly displays an inverse power law

$$
P(t) \equiv \sum_{n=1}^{L}\left|\psi_{n}(t)\right|^{2} \sim t^{-\alpha} .
$$

The best fit to the numerical data yields $\alpha=0.55 \pm 0.05$ in accordance with Eq. (11).

For a further test of the validity of Eq. (1) we now turn to the Fibonacci model, where $D_{0}^{E}$ can be varied. Here the potential $V_{n}$ only takes on two values $\pm V(V \neq 0)$ that are arranged in a Fibonacci sequence [19]. It was shown that the spectrum is a Cantor set with fractal dimension $D_{0}^{E}$ depending on $V$ 19. In Fig. 2(a) we report some of our numerical results for $P(t)$. Again we find a power-law decay $P(t) \sim t^{-\alpha}$, where the exponent depends on the potential strength $V$. The exponents 
$\alpha$ extracted for various $V$ are compared with the corresponding fractal dimension $D_{0}^{E}$ in Fig. 2(b) and confirm the validity of Eq. (11).

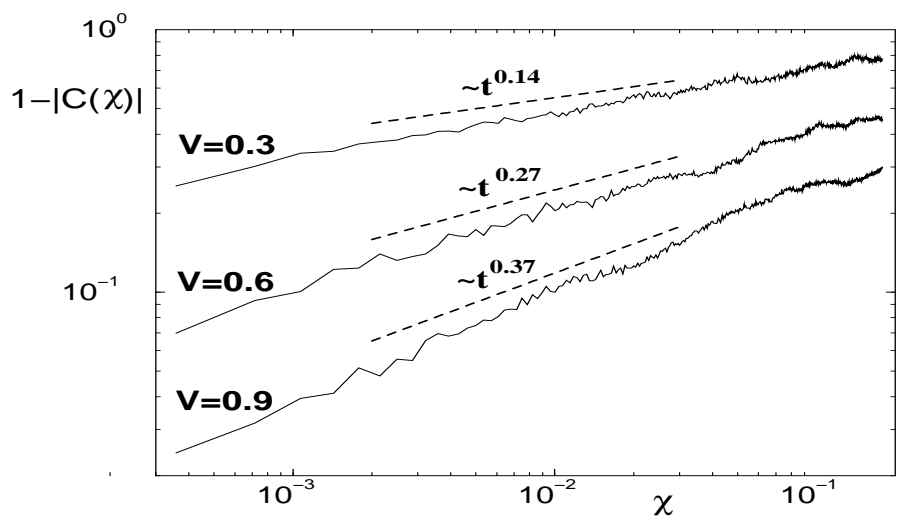

FIG. 4. The scattering autocorrelation function Eq. (8) (plotted as $1-|C(\chi)|)$ for some representative $V$ values of the Fibonacci model compared to the theoretical expectation (dashed line). In these calculations the sample has length $L=10946$ and is attached to one lead. The phase of the corresponding scattering matrix $S(E)=e^{i \Phi(E)}$ was calculated with the help of an iteration relation developed in Ref. 26.

We now want to give a general argument for the validity of Eq. (1). The effect of an open edge for the system described by Eq. (3) can be simulated by adding the imaginary shift $i$ to the first diagonal element of the Hamiltonian matrix [24]. Denoting our quasi-periodic tight-binding Hamiltonian by $H_{L}$, this approach yields an effective Hamiltonian

$$
\mathcal{H}_{e f f}=H_{L}-i \vec{e} \bigotimes \vec{e}
$$

where $\vec{e}=(1,0, \ldots, 0)^{T}$ is an $L$-dimensional vector that describes at which site we impose the absorbing boundary condition. The eigenenergies of the effective Hamiltonian are complex $\mathcal{E}_{k}=E_{k}-i \Gamma_{k} / 2$ leading to the decay of the survival probability $P(t)$. When the on-site potential fulfills $\left|V_{n}\right|>1$ the imaginary shift can be considered as a small perturbation of the Hamiltonian of the closed system. In this case according to the perturbation theory, $\Gamma_{k} \sim\left|\psi_{1}^{k}\right|^{2}$ holds, where $\psi_{n}^{k}$ is an eigenstate of the closed system with energy $E_{k}$. The survival probability is then given by $P(t) \simeq \sum_{k}\left|c_{k}\right|^{2} e^{-\Gamma_{k} t}$, where $c_{k}$ are overlapping elements of the initial state with the eigenstates $\psi_{n}^{k}$. Choosing the initial state to be concentrated at site $n=1$, we have $\left|c_{k}\right|^{2}=\left|\psi_{1}^{k}\right|^{2} \sim \Gamma_{k}$. Using this and converting the sum into an integral we obtain:

$$
P(t) \sim \int \Gamma \mathcal{P}(\Gamma) e^{-\Gamma t} d \Gamma,
$$

where $\mathcal{P}(\Gamma)$ is the resonance width distribution. In Ref. 25] it was shown that $\mathcal{P}(\Gamma) \sim \Gamma^{-\left(1+D_{0}^{E}\right)}$ for small $\Gamma$. Inserting this expression into Eq. (7) one finds the asymptotic power-law decay stated in Eq. (11). In order to check the validity of the perturbative arguments we numerically calculate the resonance widths $\Gamma_{k}$ and the overlapping elements $c_{k}$ for the Harper and Fibonacci models. As can be seen from Fig. 3 the prediction $\left|c_{k}\right|^{2} \sim \Gamma_{k}$ of the perturbation theory holds even for small $V$.

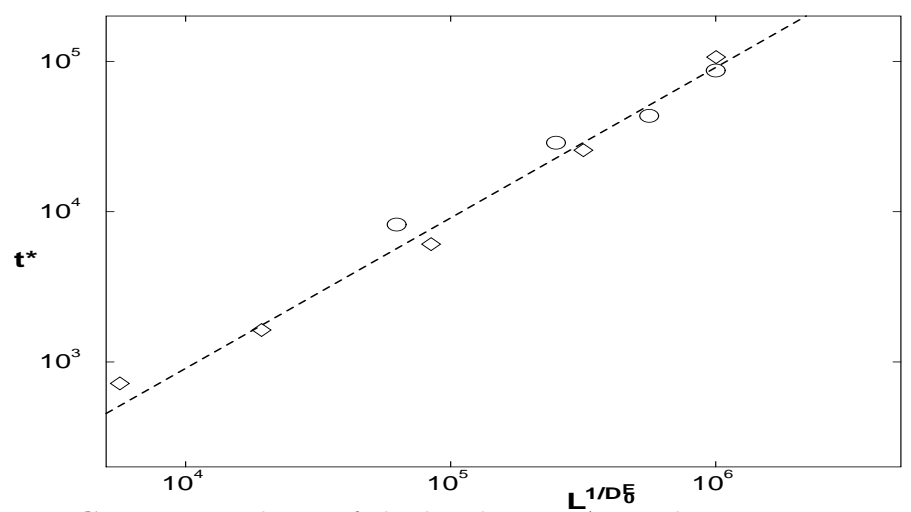

FIG. 5. Dependence of the break-time $t^{*}$, on the system size $L$ and the box-counting dimension $D_{0}^{E}$. (०) refer to the Harper model at $\lambda=2$ and various $L$ 's while $(\diamond)$ refer to the Fibonacci model with various $V$ 's and $L=2000$. The dashed line has slope 1 and corresponds to the theoretical expectation Eq. (2).

An immediate consequence of Eq. (11) is that the scattering matrix autocorrelation function $C(\chi) \equiv<$ $S(E)^{\dagger} S(E+\chi)>_{E}$, decays in the form of a power law. In particular, using the relation between the survival probability $P(t)$ and $C(\chi)[13]$, we obtain

$$
1-C(\chi) \sim \chi \int d t P(t) \exp (-i t \chi) \sim \chi^{\alpha}, \quad \chi \ll 1
$$

Equation (8) is in contrast to the Lorentzian form of $C(\chi)$ predicted by RMT for chaotic/ballistic systems [27. Comparison of $C(\chi)$ for various $V$-values of the Fibonacci model with the theoretical prediction Eq. (8) in Fig. 4 shows a nice agreement and provides an additional check for the validity of Eq. (11).

For finite samples the power-law decay of $P(t)$ (Eq. (11)) holds up to a break time $t^{*}$, beyond which it turns into an exponential decay. The rate of the latter is determined by the smallest resonance width $\Gamma_{\min }$ and thus $t^{*} \sim 1 / \Gamma_{\min }$. An estimation for $t^{*}$ can be derived as follows. Imposing the normalization condition for the resonance width distribution and assuming that the power law $\mathcal{P}(\Gamma) \sim \Gamma^{-\left(1+D_{0}^{E}\right)}$ is valid for $\Gamma \geq \Gamma_{\min }$, i.e $\int_{\Gamma_{\text {min }}}^{\infty} d \Gamma \mathcal{P}(\Gamma)=L$, we obtain Eq. (2). This prediction is verified numerically in Fig. 5 where we defined $t^{*}$ as the time where $P(t)$ deviates by $5 \%$ from the powerlaw decay. We want to point out that the increase of $t^{*}$ for decreasing $D_{0}^{E}$ is consistent with the enlargement of the interval where $\left|c_{k}\right|^{2} \sim \Gamma_{k}$ holds and its shift towards smaller values (notice the change of the axes scales in Fig. 3(b)-(d)). 
In summary we find that systems with a fractal spectrum show a power-law decay of the survival probability $P(t) \sim t^{-\left(1-D_{0}^{E}\right)}$. For the finite systems of size $L$, this decay can be observed up to a time scale $t^{*} \sim L^{1 / D_{0}^{E}}$ beyond which an exponential decay sets in. Our predictions should be observable in propagation experiments with electrons or classical waves in quasi-periodic superlattices or dielectric multilayers 28]. Moreover the connection between $\alpha, \gamma$ and $D_{0}^{E}$ provides a new possibility for experimental measurements of the fractal dimension $D_{0}^{E}$ by studying current-relaxation processes.

We thank L. Hufnagel, for useful discussions. (T.K) thanks U. Smilansky for initiating his interest in quantum scattering.

[1] C. Mahaux and H. A. Weidenmüller, "Shell Model Approach in Nuclear Reactions", (North-Holland, Amsterdam), (1969); I. Rotter, Rep. Prog. Phys. 54, 635 (1991).

[2] Atomic Spectra and Collisions in External Fields, M. H. Nayfeh et. al., eds. (Plenum, New York), Vol. 2 (1989).

[3] P. Gaspard, in "Quantum Chaos", Proceedings of E. Fermi Summer School 1991, G. Casati et. al., eds. (North-Holland) 307.

[4] P. W. Brouwer, K. M. Frahm, C. W. Beenakker, Phys. Rev. Lett. 78, 4737 (1997); M. Titov and C. Beenakker, cond-mat/ 0005042 (2000); M. Titov and Yan V. Fyodorov, Phys. Rev. B 61, R2444 (2000).

[5] T. Kottos, U. Smilansky, J. Fortuny, G. Nesti, Radio Science, 34 747, (1999); Tsampikos Kottos and Uzy Smilansky, Phys. Rev. Lett., 85 968, (2000).

[6] A. Z. Genack, P. Sebbah, M. Stoytchev and B. A. van Tiggelen, Phys. Rev. Lett., 82715 (1999); J. Stein, H.J. Stöckmann, and U. Stoffregen, Phys. Rev. Lett. 75, 53 (1995); Ulrich Kuhl, Mikrowellenuntersuchungen an eindimensionalen Streusystemen und in zweidimensionalen Kavitäten, Ulrich Kuhl, (Ph.D. Thesis, PhilippsUniversität Marburg, July 1998).

[7] B. L. Altshuler, V. E. Kravtsov, I. V. Lerner, Pisma Zh. Eksp. Teor. Fiz. 45, 160 (1987) [ JETP Lett. 45, 199 (1987)]; B. L. Altshuler, V. N. Prigodin, Zh. Eksp. Teor. Fiz. 95, 348 (1989) [ Sov. Phys. JETP 68, 409 (1980)]

[8] B. A. Muzykantskii and D. E. Khmelnitskii, Phys. Rev. B 51, 5480 (1995).

[9] A. D. Mirlin, Phys. Rep. 326, 259 (2000).

[10] V. I. Fal'ko and K. B. Efetov, Phys. Rev. B 52, 17413 (1995); V. I. Fal'ko and K. B. Efetov, Europhys. Lett. 32, 627 (1995)

[11] I. E. Smolyarenko and B. L. Altshuler, Phys. Rev. B 55, 10451 (1997).
[12] D. V. Savin and V. V. Sokolov, Phys. Rev. E 56, R4911 (1997); M. Glück, A. R. Kolovsky and H. J. Korsch, nlin.CD/0005065.

[13] F. M. Dittes, Phys. Reports 339, 215 (2000).

[14] G. Casati, G. Maspero, and D. L. Shepelyansky, Phys. Rev Lett. 82, 524 (1999); ibid, 84, 4088 (2000).

[15] G. Casati, I. Guarneri, and G. Maspero, Phys. Rev Lett. 84, 63 (2000); L. Hufnagel, R. Ketzmerick, M. Weiss, cond-mat/0009010.

[16] T. Geisel, R. Ketzmerick and G. Petschel, Phys. Rev. Lett. 66, 1651 (1991); T. Geisel, R. Ketzmerick and G. Petschel, in Quantum Chaos; Between Order and Disorder, J. Casati and B. Chirikov, eds. (Cambridge) 634-659.

[17] C. E. Porter, Statistical Theory of Spectral Fluctuations, Academic Press, New York (1965); B. L. Altshuler and B. I. Shklovskii, Zh. Eksp. Teor. Fiz. 91, 220 (1986) [Sov. Phys. JETP 64, 127 (1986)].

[18] P. G. Harper, Proc. Roy. Soc. Lond. A68, 874 (1955); S. Aubry, G. Andre, Ann. Israel Phys. Soc. 3, 133 (1980); S. N. Evangelou, J.-L. Pichard, Phys. Rev. Lett. 84, 1643 (2000); F. Piéchon, Phys. Rev. Lett., 76 4372, (1996).

[19] D. Shechtman, I. Blech, D. Gratias, and J. V. Cahn, Phys. Rev. Lett. 53, 1951 (1984); M. Kohmoto, L. P. Kadanoff and G. Tang, Phys. Rev. Lett. 501870 (1983); J. M. Luck and D. Petritis, J. Stat. Phys. 42, 289 (1986); J. Bellisard, B. Iochum, E. Scoppola, and D. Testard, Comm. Math. Phys. 125, 527 (1989).

[20] P. Leboeuf, J. Kurchan, M. Feingold, and D. P. Arovas, Phys. Rev. Lett. 65, 3076 (1990); T. Geisel, R. Ketzmerick and G. Petschel, Phys. Rev. Lett. 67, 3635 (1991); R. Artuso, F. Borgonovi, I. Guarneri, L. Rebuzzini, and G. Casati, Phys. Rev. Lett. 69, 3302 (1992);

[21] Our results valid also for the case of a semi-infinite lattice with one absorbing boundary.

[22] M. Weiss, Tsampikos Kottos, and T. Geisel, condmat/0005339; W. H. Press and S. A. Teukolsky et al., "Numerical Recipes in Fortran 90", (Cambridge University Press), (1996);

[23] Y. Last, Commun. Math. Phys. 164, 421 (1994); R. Ketzmerick, K. Kruse, F. Steinbach, and T. Geisel,Phys. Rev. B 58, 9881, (1998).

[24] Y. V. Fyodorov, H-J Sommers, J. Math. Phys. 38, 1918 (1997); M. Terraneo, and I. Guarneri, Eur. Phys. J. B 18, 303 (2000).

[25] F. Steinbach, A. Ossipov, Tsampikos Kottos, and T. Geisel, Phys. Rev. Lett. 85, 4426 (2000).

[26] A. Ossipov, Tsampikos Kottos, and Theo Geisel, Phys. Rev. B, 61 11411, (2000).

[27] R. Blümel and U. Smilansky, Phys. Rev. Lett., 60477 (1988).

[28] U. Kuhl and H.-J. Stöckmann, Phys. Rev. Lett. 80, 3232 (1998). 